\title{
BMJ Open Prospective Investigation of Pesticide Applicators' Health (PIPAH) study: a cohort study of professional pesticide users in Great Britain
}

\author{
Anne-Helen Harding, ${ }^{1}$ David Fox, ${ }^{1}$ Yiqun Chen, ${ }^{1}$ Neil Pearce, ${ }^{2}$ David Fishwick, ${ }^{1}$ \\ Gillian Frost ${ }^{1}$
}

To cite: Harding A-H, Fox D, Chen Y, et al. Prospective Investigation of Pesticide Applicators' Health (PIPAH) study: a cohort study of professional pesticide users in Great Britain. BMJ Open 2017;7:e018212. doi:10.1136/ bmjopen-2017-018212

- Prepublication history for this paper is available online. To view these files, please visit the journal online (http://dx.doi. org/10.1136/bmjopen-2017018212).

Received 13 June 2017 Revised 28 August 2017 Accepted 29 August 2017

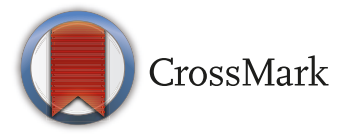

${ }^{1}$ Health and Safety Laboratory, Health and Safety Executive, Buxton, UK

${ }^{2}$ Department of Noncommunicable Disease

Epidemiology, London School of Hygiene and Tropical Medicine, London, UK

Correspondence to Dr Anne-Helen Harding; anne-helen.harding@hsl.gsi. gov.uk

\section{ABSTRACT}

Purpose The purpose of the study is to monitor the exposure and health of workers in Great Britain who use pesticides as a part of their job, and to gain a better understanding of the relationship between long-term exposure to pesticides and health.

Participants Study participants are professional pesticide users who are certified in the safe use of pesticides or who were born before 1965 and apply pesticides under 'grandfather rights'. Overall response rate was $20 \%$; participants are mostly male (98\%) and the average age is 54 years, ranging from 17 to over 80 years.

Findings to date Participants have completed a baseline general questionnaire and three follow-up questionnaires on the use of pesticides. These data will enable investigations into the relationship between occupational pesticide exposure and health outcomes taking into account non-occupational confounding factors.

Future plans There is no set end date for data collection. Recruitment into the cohort will continue, and for the foreseeable future there will be annual pesticide use questionnaires and five yearly follow-up general questionnaires. The intention is to validate the pesticide use questionnaire, and to develop a crop/job exposure matrix (C/JEM) which can be updated regularly. This $\mathrm{C} /$ JEM will be able to look at general categories of pesticide, such as insecticides, structurally related pesticides, such as organochlorines, or individual active ingredients. Data collected on use of personal protective equipment and method of application will provide information on how potential exposure to pesticide during application may have been modified. The study will be able to estimate changes in individual pesticide use over time, and to examine the associations between pesticide use and both baseline and long-term health outcomes. The cohort members will be linked to national databases for notification of hospital episode statistics, cancer incidence and mortality for follow-up of health outcomes.

\section{INTRODUCTION}

Pesticides and biocides are designed and developed to be toxic to specific plant or animal pests. There is a long history of their use; compared with the simple treatments
Strengths and limitations of this study

- Breadth of data collected comprising comprehensive demographic, lifestyle and socioeconomic data, general history of pesticide use and detailed prospective information on pesticide use.

- Objective assessment of health by flagging the cohort for notification of hospital admissions, and cancer and death registrations.

- Repeat assessments of pesticide use, potential confounding factors and self-reported health outcomes planned.

- The lack of objective exposure measurement which may result in exposure misclassification.

- There may be some participation bias but this is unlikely to be large.

used in earlier times, such as arsenic, the pesticides developed for use today have active ingredients which are usually highly effective against particular pests. ${ }^{1}$ Pesticides are almost as diverse as the pests they target, but they should have all been tested for their efficacy and for their effect on the environment and on non-target organisms, including humans. In Great Britain (GB), all pesticide products must be authorised by the Chemicals Regulation Division of the Health and Safety Executive (HSE) before they can be sold, distributed, stored or used (https://www. gov.uk/pesticide-approval). Despite their potential for harm, pesticides are widely used because of the important role they play in improving public health and protecting food supplies. $^{2}$

Many epidemiological studies have investigated the association between the use of pesticides and adverse long-term health effects. However, ascertaining the causes of disease within pesticide-exposed populations is complex because of the multifactorial nature of many diseases and the presence of 
other potential risk factors. ${ }^{3}$ Genetic susceptibility, lifestyle factors and environmental exposures may all affect risk of disease, either independently or through interactions with one another. In addition, the term pesticide covers a wide range and diverse group of chemicals or products. When investigating the health risks associated with pesticide exposure, identifying the cause of disease is complicated by the fact that individuals are often exposed to several types of pesticide, which may have different modes of action, and they may also be exposed to other potential risk factors, such as fuel and exhaust fumes, solvents, ultraviolet radiation, organic and inorganic dusts, and animal pathogens.

Many epidemiological studies have researched occupational groups who use or are exposed to pesticides as part of their daily activities. ${ }^{3}$ Workers for these studies were recruited from the agricultural, horticultural, forestry, amenity or pesticide manufacturing sectors, because they are likely to regularly handle one or more types of pesticide during the course of their work. The body of evidence on the association between exposure to pesticides and disease provides a mixed picture, and the meta-analyses undertaken often report significant heterogeneity between studies. ${ }^{3-5}$ This heterogeneity may be attributable to a variety of factors, including differences in exposure assessment, disease status ascertainment and study design, and to confounding.

A recent systematic review of epidemiological studies linking pesticide exposure to health effects was commissioned by the European Food Safety Authority ${ }^{3}$ and published in 2013. They reviewed over 600 articles published between 2006 and 2012, which included nearly 6500 different analyses of the association between pesticide exposure and health outcomes. The majority of studies $(55 \%)$ investigated occupational exposures. The health outcomes identified in the review were assigned to 23 major disease categories; the most common outcomes reported in the studies reviewed were cancer (29\%), child health $(15 \%)$, reproductive diseases $(11 \%)$ and neurological conditions $(11 \%)$. However, despite the very large amount of data available, encompassing all adverse health outcomes identified in the literature review, the authors report that findings were inconclusive for the majority of health outcomes studied. Some of the strongest evidence linking pesticide exposure to disease was found for certain cancers, particularly childhood cancers, and neurological conditions. Other health effects, including asthma, allergies, obesity and endocrine disorders, also showed an increased risk. ${ }^{3}$

Mostafalou and Abdollahi ${ }^{2}$ also published a review of pesticides and chronic diseases in 2013. Their comprehensive review covered publications between 1975 and 2013, and it included a wide range of diseases. However, the review was not systematic and the authors did not focus on epidemiological studies, but instead they gave an insight into possible causal molecular mechanisms. The major disease categories included in this review were cancer, cardiovascular disease, diabetes, developmental and reproductive disorders, kidney disease, neurological conditions, respiratory disease and autoimmune disease. Pesticide exposure has been linked with genetic damage and epigenetic changes, both of which are associated with cancer. Epigenetic changes have also been implicated in neurological conditions, diabetes, ageing, chronic kidney disease and atherosclerosis. Whether the epigenetic changes in these diseases are related to pesticide exposure or are markers of exposure ${ }^{6}$ has not been established. Some pesticides have been shown to impair mitochondrial function, increase oxidative and endoplasmic reticular stress, affect the unfolded protein response and protein degradation, or to be endocrine disruptors. ${ }^{2}$ These mechanisms have all been implicated in the development of chronic disease but further research is needed to confirm whether exposure to particular pesticides is on the causal pathway.

The body of evidence reporting on the association between pesticides and ill health is largely inconclusive, and despite the large number of studies in this area, more information is needed on the potential adverse effects of pesticide exposure on chronic disease risk. In 1998, the GB HSE established the Pesticide Users' Health Study (PUHS), a prospective study of nearly 67000 professional pesticide users. ${ }^{7}$ Their aim was to monitor the long-term health of pesticide users who are potentially exposed to low levels of pesticide. Individuals obtaining certificates of competence in the safe use of pesticides were invited to take part in the study, and the cohort was followed up for long-term health outcomes through national registers for notification of cancer and death registrations. Cancer incidence overall was lower, and standardised mortality ratios for all causes and all cancers were significantly lower in these pesticide users than in the general population. Incidence of multiple myeloma, cancer of the testes and non-melanoma skin cancer was higher than expected in men, and in women non-melanoma skin cancer was higher. None of the standardised mortality ratios for specific causes of death, including respiratory and neurological diseases, were higher than expected. However, being certified for the safe use of pesticides may not necessarily indicate using pesticides regularly and there is no active follow-up of the study participants to monitor the changes in their pesticide use. Furthermore, only basic demographic information was available for this cohort so interpretation of the findings in terms of causal relationships is not possible.

In 2013, HSE established the Prospective Investigation of Pesticide Applicators' Health (PIPAH) study. Professional pesticide users were enrolled into the PIPAH study, and baseline data were collected on self-reported health and potential risk factors. The PIPAH study is smaller than the PUHS, but it is collecting the detailed information which is lacking in the PUHS, such as information on pesticide exposure at product level and potential confounding factors. The PIPAH study's aims are similar to those of the PUHS: the overall aims are to monitor the long-term health of workers in GB who use pesticides as 


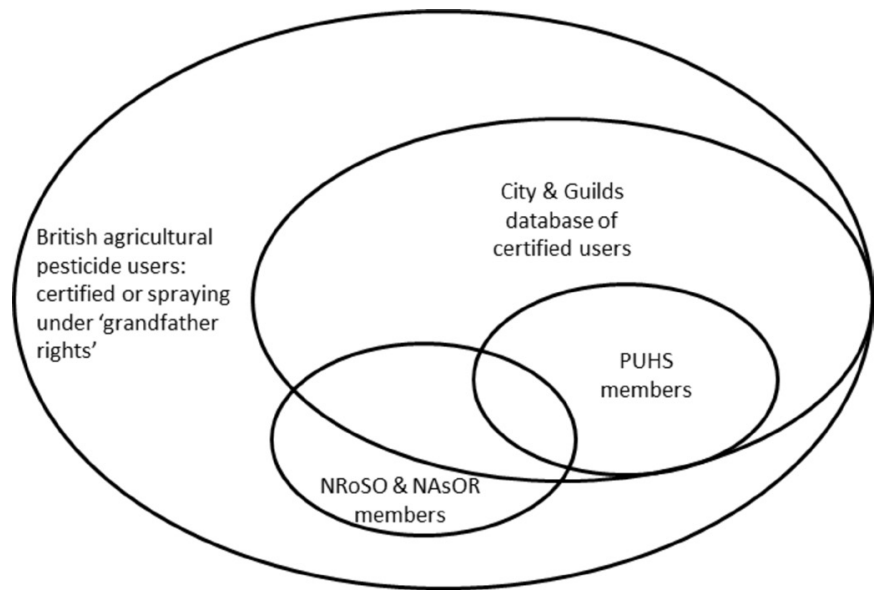

Figure 1 The relationship between the registers of pesticide users, the PUHS and the target population. NAsOR, National Amenity Sprayer Operators' Register; NRoSO, National Register of Sprayer Operators; PUHS, Pesticide Users' Health Study.

a part of their job, and to gain a better understanding of the relationship between long-term exposure to pesticides and health. A profile of the cohort, including a detailed description of the cohort design, methods and baseline population characteristics, is presented in this paper.

\section{COHORT DESCRIPTION}

\section{Study design}

The study is a prospective cohort study, which includes a baseline cross-sectional survey at recruitment and ongoing follow-up of study participants' pesticide use and health outcomes. Data will be collected by conducting periodical surveys and through linkage to administrative health records.

\section{Target population and sampling frame}

The target population for the PIPAH study is men and women in GB who apply pesticides as a part of their job. Individuals belonging to this population were identified through a number of registers. Until November 2015, individuals applying pesticides on a professional basis in GB were required to be certified in the safe use of pesticides, unless they were born before 1965 in which case they could apply pesticides under 'grandfather rights'. From November 2015, 'grandfather rights' were revoked and everyone wishing to apply a professional pesticide must now be certified. City \& Guilds (http://www.cityandguilds.com/) offers the necessary training and keeps a register of all individuals who hold certificates of competence. Members of the PUHS were recruited from this training register. In addition to this register, City \& Guilds maintains the National Register of Sprayer Operators (NRoSO, https://www.nroso.org.uk/) and previously also the National Amenity Sprayer Operators' Register (NAsOR). These are central registers of sprayer operators in GB who use continuing professional development as a means of ensuring ongoing training. The relationship between the registers, the PUHS and the target population is shown in figure 1 . The members of NRoSO and NAsOR, and the subgroup of the PUHS who responded to a study questionnaire in 2004-2006, were invited to take part in the PIPAH study.

\section{Recruitment and response rates}

Enrolment in the baseline phase of the PIPAH study was carried out in two stages: NRoSO and NAsOR members were recruited during the first half of 2013, and the subset of PUHS members was recruited in early 2014. Approximately 21000 members of NRoSO and NAsOR, and around 7500 PUHS members were sent a survey pack inviting them to participate in the PIPAH study. The packs contained an information leaflet, consent form, questionnaire and a postage paid envelope for the return of completed questionnaires. During the first stage, when recruiting members of NRoSO and NAsOR, a reminder postcard was sent to everyone a week after the first invitation pack. During the second stage, when recruiting members of the PUHS, a full survey pack was sent to everyone who had not responded 4 weeks after the first invitation. In addition to the baseline phase, all new members of NRoSO are invited to take part in the PIPAH study in an ongoing rolling recruitment programme. Recruitment activities have resulted in the collection of baseline data from $5731 \mathrm{~GB}$-based pesticide users by

Table 1 Recruitment data and overall response rates

\begin{tabular}{|c|c|c|c|c|c|c|c|}
\hline & \multicolumn{2}{|c|}{ Responders } & \multicolumn{2}{|c|}{ Non-responders } & \multicolumn{2}{|l|}{ All } & \multirow{2}{*}{$\begin{array}{l}\text { Response rate } \\
\%\end{array}$} \\
\hline & $\mathbf{N}$ & $\%$ & $\mathbf{N}$ & $\%$ & $\mathbf{N}$ & $\%$ & \\
\hline \multicolumn{8}{|l|}{ Recruitment phase } \\
\hline PUHS & 1676 & 29.2 & 5921 & 25.7 & 7597 & 26.5 & 22.1 \\
\hline Subtotal & 5624 & & 23024 & 100.0 & 28648 & 100.0 & $19.6^{*}$ \\
\hline Total & 5731 & 100.0 & & & & & \\
\hline
\end{tabular}

${ }^{*}$ Overall response rate.

NAsOR, National Amenity Sprayer Operators' Register; NRoSO, National Register of Sprayer Operators; PUHS, Pesticide Users' Health Study. 


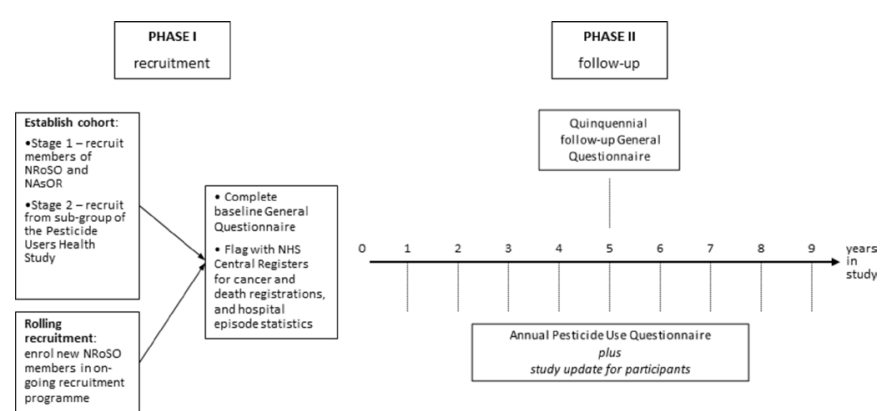

Figure 2 The PIPAH (Prospective Investigation of Pesticide Applicators' Health) study flow chart. NAsOR, National Amenity Sprayer Operators' Register; NHS, National Health Service; NRoSO, National Register of Sprayer Operators.

March 2014. Recruitment data and response rates are described in table 1 . Response rates for men and women were similar at around $20 \%$.

\section{Data collection and follow-up}

A baseline general questionnaire was developed. Where possible, questions were based on validated questions used in other cohort studies. The questionnaire was tested in face-to-face interviews with professional pesticide users before being included in the study invitation pack. On joining the study, participants completed the baseline general questionnaire which included sections on demographic, diet, lifestyle and socioeconomic factors; job history and history of pesticide use; family medical history; and self-reported ill health. It was unrealistic to gather accurate detailed lifetime pesticide use information retrospectively using a self-completion questionnaire. So the history of pesticide use questions concentrated on pesticide groups and crops which could be refined using a crop/job exposure matrix. Follow-up general questionnaires will be sent to study participants every 5 years (figure 2 ). This will provide updated information on self-reported ill health and potential risk factors. The follow-up general questionnaires will incorporate additional disease-specific question sets, for example, on respiratory health, which are relevant to this occupational cohort.

Information on pesticide exposure is collected on an annual basis. Pilot pesticide use questionnaires were sent to age-stratified random samples of 400 participants in early 2014, 2015 and 2016. The remaining participants were sent a short postcard questionnaire which requested information on the main areas of their pesticide work. The postcard questions are included in the full pilot questionnaires. After validating the pilot questionnaire, the detailed pesticide use questionnaire was sent to all study members in 2017.

The data collected in the general questionnaire and the pesticide use questionnaires are summarised in box.

Study participants will be linked with GB central registers for notification of hospital episode statistics, and cancer and death registrations. This is an efficient and effective method of following up on participants' health outcomes in the long term.

\section{BASELINE CHARACTERISTICS OF THE STUDY PARTICIPANTS}

The mean age for the cohort overall was 54 years. The mean age of the NRoSO (52 years) and NAsOR (53years) participants was similar, while the PUHS members were older (mean age 60years). Study participants are predominantly male $(98 \%)$ which reflects the male-female proportion in the sampling frame.

The highest level of education achieved is shown in table 2 . The proportion of cohort members who reported that their highest level of education was General Certificate of Secondary Education or equivalent was similar to the general population of England and Wales $(28 \%) .^{8}$ A smaller proportion of study participants had

Box Data collected by the general questionnaire and the pesticide use questionnaire

Baseline general questionnaire

$>$ Age

- Time lived on a farm and farm type

- Work history

- Crops where pesticides have been used (current and past)

- Number of years applying specific types of pesticides (eg, herbicides)

- Number of days spent applying specific types of pesticides in a typical year

- Decade when a specific type of pesticide was first used

- Use of personal protective equipment

- Use of pesticide concentrate

- Application methods

- Repair and maintenance of application/mixing equipment

- General health

- Ever been doctor diagnosed with a range of specified health conditions

- Self-report of a range of ill health symptoms

- Family medical history

- Lifestyle

- Physical activity

- Time spent outdoors and use of sun protection measures

- Diet

- Tobacco and alcohol consumption

- Circumstances

- Marital status

- Home ownership

- Qualifications

- Employment status

Follow-up pesticide use questionnaire

- Work history during the last year

- Pesticide products used in the last year

- The number of days each pesticide product was used in the last year

- The typical number of hours per day each pesticide product was used in the last year

- Whether each pesticide product was liquid, dry or a gas (added in 2016)

- Application method

- Use of personal protective equipment 
Table 2 Education, marital and employment status

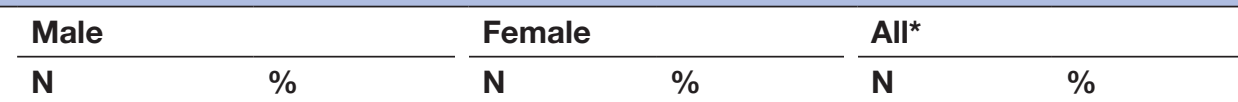

\section{Education status}

GCSE, O-level or equivalent

1339

A-level or equivalent

415

Vocational

1379

Degree or higher degree

897

No formal or other†

1363

Subtotal

5393

Missing

154

Total

5547

24.8

7.7

25.6

16.6

25.3

100.0

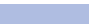

Marital status

Never married

Married

\begin{tabular}{lrlrlr}
509 & 9.5 & 18 & 15.0 & 529 & 9.6 \\
4069 & 75.7 & 64 & 53.3 & 4161 & 75.3 \\
404 & 7.5 & 17 & 14.2 & 424 & 7.7 \\
104 & 1.9 & 8 & 6.7 & 112 & 2.0 \\
288 & 5.4 & 13 & 10.8 & 303 & 5.5 \\
5374 & 100.0 & 120 & 100.0 & 5529 & 100.0 \\
173 & & 6 & & 202 & \\
5547 & & 126 & & 5731 & \\
1968 & & & & & \\
2867 & 36.2 & 59 & 48.8 & 2031 & 36.3 \\
607 & 52.7 & 42 & 34.7 & 2932 & 52.4 \\
5442 & 11.2 & 20 & 16.5 & 637 & 11.4 \\
105 & 100.0 & 121 & 100.0 & 5600 & 100.0 \\
5547 & & 5 & & 131 & \\
\hline
\end{tabular}

Living together

Widowed

Divorced/separated

Subtotal

Missing or otherł

Total

Employment status

\section{Employed}

Self-employed

Other

Subtotal

Missing

Total

*Includes 58 people missing response for sex. †No formal category includes small numbers. ‡Other category includes small numbers.

A-level, advanced level; GCSE, General Certificate of Secondary Education; O-level, ordinary level.

advanced levels (or equivalent), or a degree/higher degree than the general population (12\% and $27 \%$, respectively ${ }^{8}$ ), but $25 \%$ of the cohort had vocational qualifications and a substantial proportion had other (most likely vocationally based) qualifications. Almost three quarters of the cohort reported being married, which is substantially higher than the $47 \%$ who reported being married in the general population. ${ }^{8}$ The proportion of study participants who were divorced or separated was approximately half that in the general population $(12 \%){ }^{8}$ The majority of the cohort (52\%) described themselves as self-employed compared with nearly $15 \%$ in the population of the UK. ${ }^{9}$ Approximately $36 \%$ of the cohort reported that they were employed compared with almost $75 \%$ for England and Wales. ${ }^{8}$ These differences are likely to be attributable to the high proportion of farmers in the cohort, many of whom are self-employed. The educational, marital and employment profiles of the cohort differ from those of the general population in a number of respects but these comparisons are not adjusted for the differences in age and gender between the study participants and the general population.

\section{LIFESTYLE CHARACTERISTICS AT BASELINE}

Table 3 summarises the status of the cohort with regard to alcohol consumption and smoking status. The majority of the cohort (94\%) reported that they currently drink alcohol, which is just over nine percentage points higher than GB-based comparator statistics. ${ }^{10}$ The reported proportion of current smokers in the cohort at $9 \%$ is around half of the estimated $19 \%$ smoking rate in England, ${ }^{11}$ although about $28 \%$ report that they have smoked in the past. These comparisons between the cohort and population-wide statistics are not adjusted for age or sex, but the statistics suggest that the study participants differ from the general population, particularly with respect to their smoking status. 
Table 3 Alcohol consumption and smoking status

\begin{tabular}{|c|c|c|c|c|c|c|}
\hline & \multicolumn{2}{|l|}{ Male } & \multicolumn{2}{|c|}{ Female } & \multicolumn{2}{|l|}{$\mathrm{All}^{*}$} \\
\hline & $\mathbf{N}$ & $\%$ & $\mathbf{N}$ & $\%$ & $\mathbf{N}$ & $\%$ \\
\hline \multicolumn{7}{|c|}{ Alcohol consumption status } \\
\hline Former drinker & 189 & 3.6 & 7 & 5.9 & 196 & 3.6 \\
\hline Current drinker & 4988 & 94.2 & 108 & 90.8 & 5132 & 94.2 \\
\hline Missing & 252 & & 7 & & 281 & \\
\hline Total & 5547 & & 126 & & 5731 & \\
\hline \multicolumn{7}{|l|}{ Smoking status } \\
\hline Never smoked & 3216 & 63.4 & 77 & 67.5 & 3311 & 63.4 \\
\hline Former smoker & 1403 & 27.7 & 25 & 21.9 & 1439 & 27.6 \\
\hline Total & 5547 & & 126 & & 5731 & \\
\hline
\end{tabular}

*Includes 58 people missing response for sex.

Some of the important components of diet are summarised in figure 3. Altogether, 9.5\% ( $\mathrm{n}=290)$ reported eating five or more portions of fruit and vegetables per day, compared with $30 \%$ of adults aged 19-64years in GB who meet the government minimum target of five portions of fruit and vegetables per day. ${ }^{12}$ Overall, 94\% $(n=4962)$ of the cohort reported eating one portion of red or processed meat less than once a day and $62 \%(\mathrm{n}=3508)$ reported eating oily fish at least once a week. The GB nutritional guidelines recommend that adults do not eat more than one portion of red or processed meat per day, and that they should eat two portions of fish per week. The latter should include one portion of oily fish.

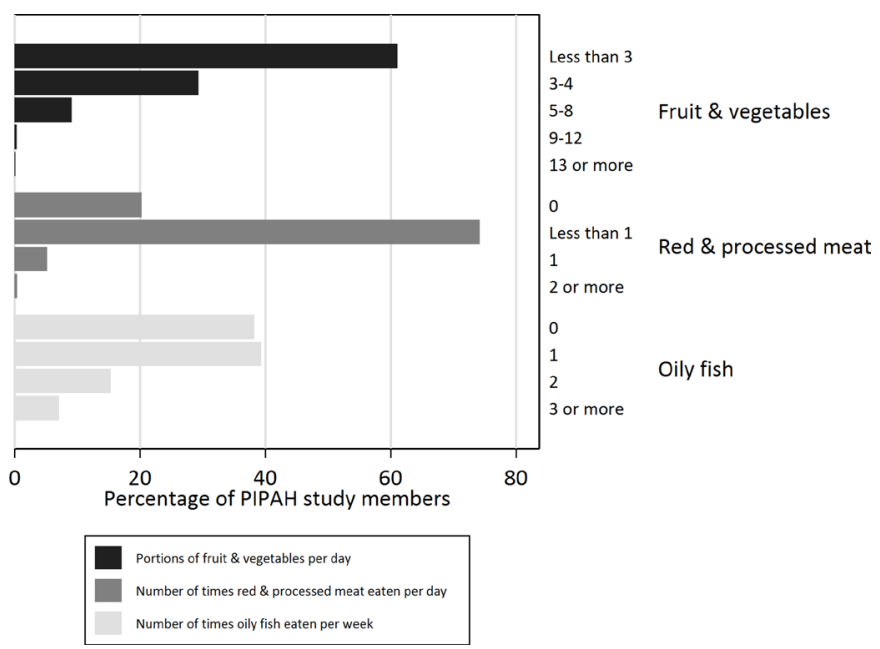

Figure 3 Frequency of fruit and vegetables, red and processed meat, and oily fish consumption. PIPAH, Prospective Investigation of Pesticide Applicators' Health.

\section{MAIN AREAS OF PESTICIDE WORK}

The men and women who enrolled in the PIPAH study applied pesticides in a wide range of work areas. Cereals, oilseeds and grassland/fodder crops currently involve the highest proportions of cohort members $(65 \%, 48 \%$ and $40 \%$, respectively) all of which belong to the agricultural sector (figure 4). Smaller proportions of participants were involved in other areas of pesticide work such as the amenity sector $(9.6 \%)$, forestry $(3.4 \%)$ and orchard crops $(2.8 \%)$. There were substantial increases in the proportions working in cereals, oilseeds and grassland/fodder crops compared with their past areas of work and there was a notable reduction in the proportion of the cohort reporting involvement in potato-related pesticide work.

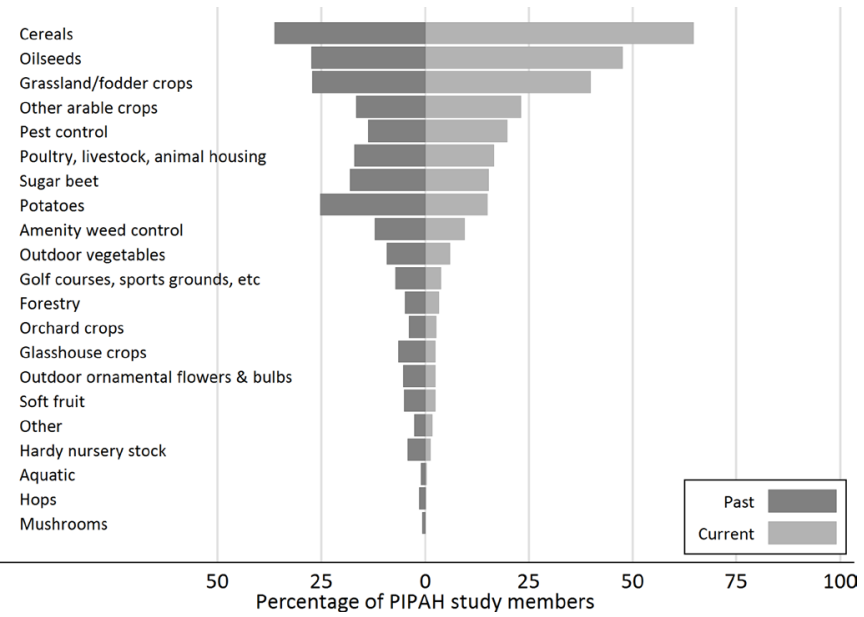

Figure 4 Past and current areas of pesticide work. PIPAH, Prospective Investigation of Pesticide Applicators' Health. 

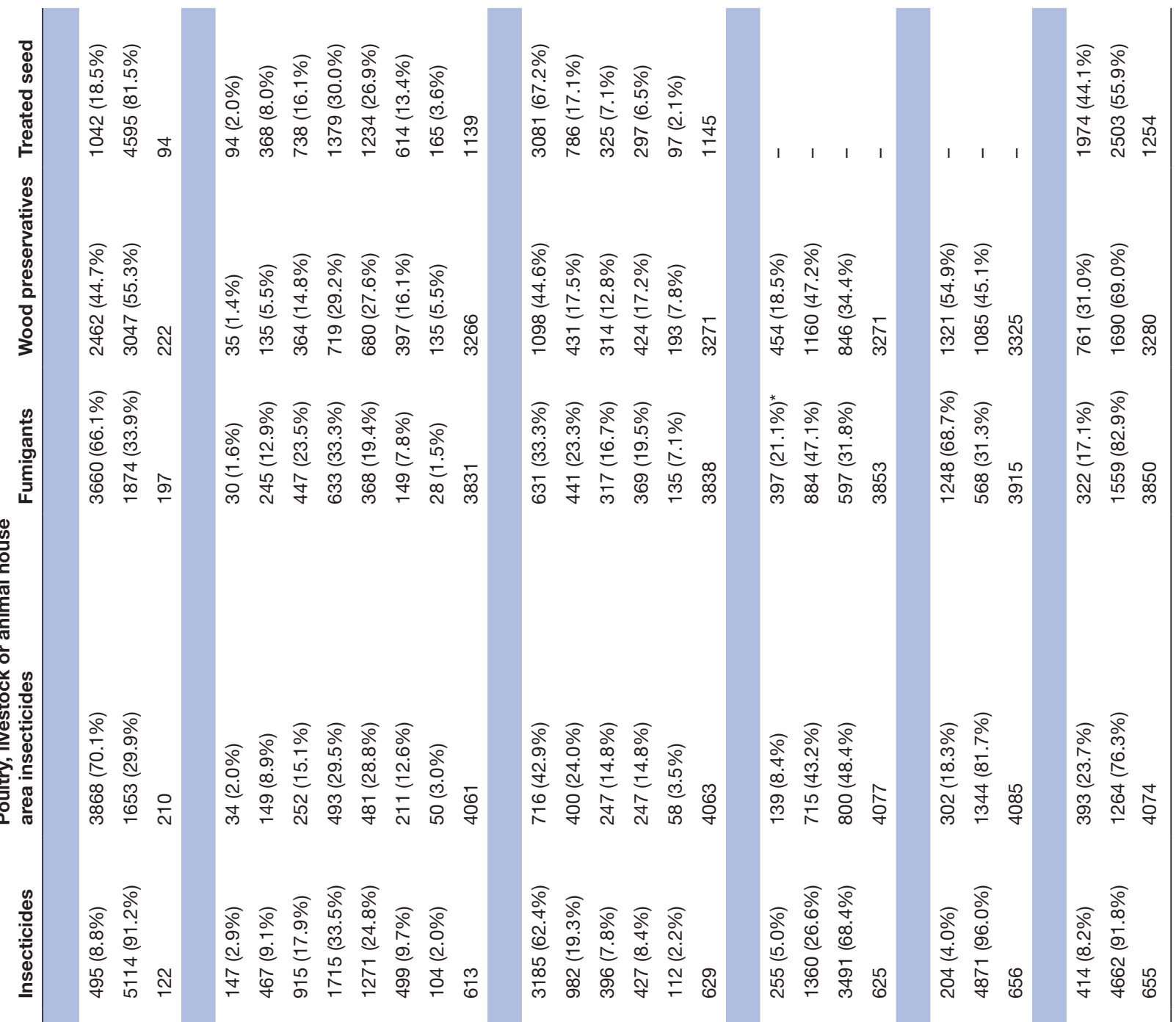

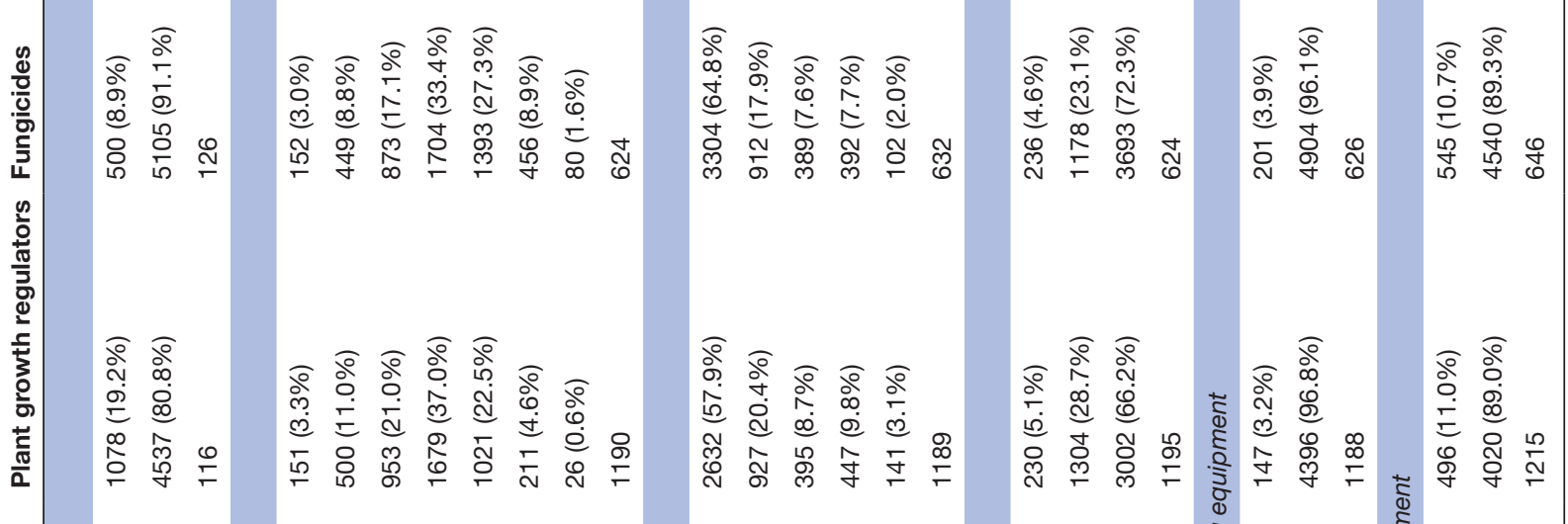

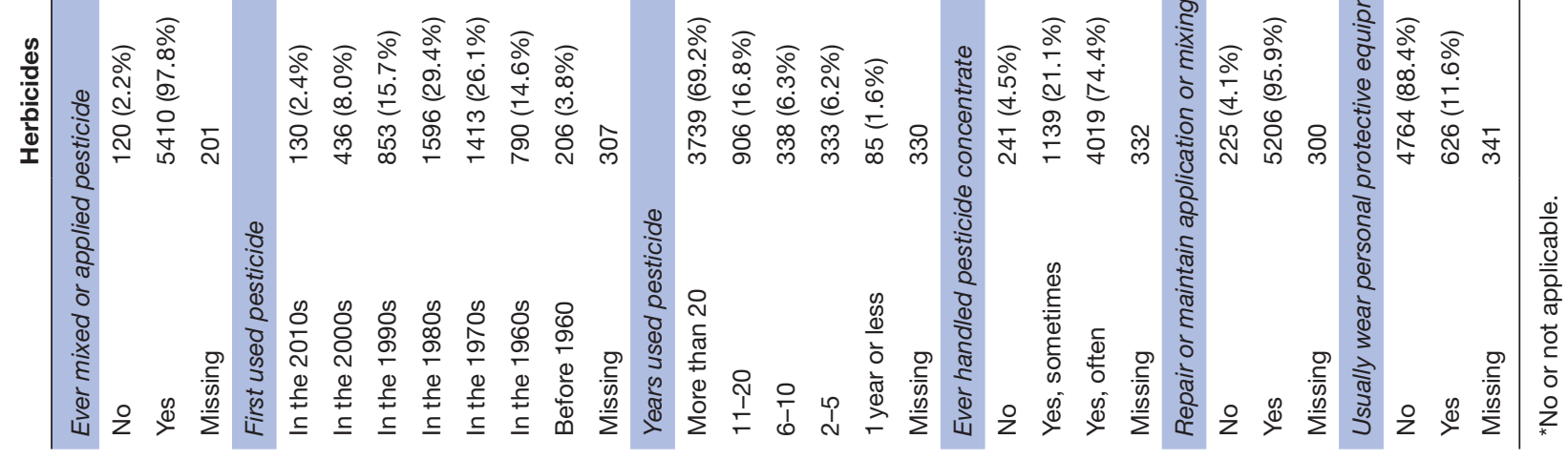




\section{HISTORY OF WORKING WITH PESTICIDES}

Table 4 summarises the participants' history of working with pesticides before joining the study. The large majority of participants (over 80\%) have worked with herbicides, plant growth regulators, fungicides, insecticides and treated seed, while smaller percentages have worked with poultry, livestock and animal house area insecticides, fumigants and wood preservatives. The largest proportion of participants began working with pesticides during the 1970s, 1980s and 1990s, and across all of the pesticide groups most participants had worked with pesticides for more than 10 years. This distribution reflects the age structure of the cohort. The majority of participants have handled pesticide concentrate, which has previously been observed to increase the risk of reporting 'ill health'. ${ }^{13}$ Except when working with herbicides, most participants used personal protective equipment when mixing, handling or applying pesticides; only $12 \%$ reported using personal protective equipment when working with herbicides.

\section{SELF-REPORTED HEALTH}

Cohort members were asked to report any doctor-diagnosed health outcomes. The categories of disease included were respiratory, neurological, circulatory, ophthalmic, dermatological, musculoskeletal, metabolic and other conditions including glandular fever, lead poisoning, pesticide poisoning, ulcerative colitis or Crohn's disease, injury (excluding head injury) from farm machinery and head injury requiring medical attention. The lifetime prevalence was greater than $10 \%$ for work-related musculoskeletal injuries (23.5\%), high blood pressure (14.8\%) and asthma (10.4\%). Injury from farm machinery $(9.9 \%)$ and head injury (7.1\%) were also common in this occupational group. Health outcomes with a lifetime prevalence of less than $1 \%$ included Alzheimer's disease, chronic obstructive pulmonary disease, chronic kidney infections, epilepsy, multiple sclerosis and Parkinson's disease.

\section{STRENGTHS AND LIMITATIONS}

A key strength of the PIPAH study is the breadth of the data, including comprehensive demographic, lifestyle and socioeconomic data and detailed information about use of pesticides, which have been and will be collected from a large group $(>5000)$ of clearly defined professional pesticide users. These data, in combination with the data collected about past and current health conditions and the prospective design of the study, will allow the research team to conduct novel analyses exploring the relationships between pesticide exposure and health outcomes.

Another important strength of the study design is the planned repeated assessment of pesticide use, basic demographic and lifestyle factors, and self-reported health outcomes. Keeping this information current will improve assessment of exposure in future analyses, and repeat assessments will provide opportunities to add new question sets into the questionnaires. Flagging cohort members for notification of hospital episodes, and cancer or death registrations provides a very effective objective means of following up long-term health outcomes with minimal loss to follow-up and no burden on participants.

Potential limitations of the study include the possibility of participation bias due to self-selective decisions to take part in the study. However, there is no evidence to suggest that the decision to take part in the study was influenced by participants' exposure or health. The proportions of men and women in the study sampling frame and the cohort were identical, but the age structure differed in that the cohort members were a little older.

The study relies on self-reported pesticide use rather than objective exposure measurements, which is a potential weakness. Exposure misclassification may occur, which may affect the ability of the study to investigate the real associations between pesticide exposure and health. Biological monitoring would provide a more accurate measure of the pesticide exposure actually experienced, but costs and logistics prevent this from being carried out on the full cohort. Future research investigating the health risks associated with particular pesticides and using biological monitoring to assess exposure could be undertaken on a subset of the cohort.

Acknowledgements The authors would like to thank the participants for their contribution and continuing support for the study. The authors would also like to thank City \& Guilds for their assistance in recruiting the pesticide users into the study, and for enabling active engagement with study participants at the agricultural shows. Finally, the authors would like to thank the PIPAH study team for their many and varied contributions.

Contributors DFox, GF, YC, DFishwick and AHH are all members of the study team, and were involved in the original concept and design of the study. NP contributed to the design of the study and the survey instruments. DF, GF, YC and AHH were responsible for developing the survey tools, and for data collection, analysis, interpretation and drafting of the manuscript. All the authors revised the manuscript, agreed with the findings and approved the final version.

Funding Health and Safety Executive

Disclaimer This publication and the work it describes, including any opinions and/ or conclusions expressed, are those of the authors and do not necessarily reflect HSE policy

Competing interests None declared.

Patient consent Obtained.

Ethics approval National Research Ethics Service Committee North West-Greater Manchester North (reference 12/NW/0654).

Provenance and peer review Not commissioned; externally peer reviewed.

Data sharing statement The PIPAH study team would welcome opportunities for research collaboration. For further information on accessing the anonymised research data already collected as part of this study or to contact researchers with research proposals, please contact the PIPAH study team (PIPAH@hsl.gsi.gov. uk). A copy of the baseline general questionnaire and the follow-up pesticide use questionnaire is available on the PIPAH web page (http://www.hsl.gov.uk/resources/ major-projects/pipah).

Open Access This is an Open Access article distributed in accordance with the Creative Commons Attribution Non Commercial (CC BY-NC 4.0) license, which permits others to distribute, remix, adapt, build upon this work non-commercially, and license their derivative works on different terms, provided the original work is properly cited and the use is non-commercial. See: http://creativecommons.org/ licenses/by-nc/4.0/ 
(c) Article author(s) (or their employer(s) unless otherwise stated in the text of the article) 2017. All rights reserved. No commercial use is permitted unless otherwise expressly granted.

\section{REFERENCES}

1. Unsworth J. History of pesticide use: International Union of Pure and Applied Chemistry, 2010. updated 10.05.2010. http://agrochemicals. iupac.org/index.php?option=com_sobi2\&sobi2Task=sobi2Details\& catid=3\&sobi2ld=31 (accessed 25 Jan 2010).

2. Mostafalou S, Abdollahi M. Pesticides and human chronic diseases: evidences, mechanisms, and perspectives. Toxicol Appl Pharmacol 2013;268:157-77.

3. Ntzani EE, Ntritsos G CM, Evangelou E, et al. Literature review on epidemiological studies linking exposure to pesticides and health effects. EFSA Supporting Publications 2013;10:159.

4. van der Mark M, Brouwer M, Kromhout $\mathrm{H}$, et al. Is pesticide use related to Parkinson disease? Some clues to heterogeneity in study results. Environ Health Perspect 2012;120:340-7.

5. Perrotta C, Staines A, Cocco P. Multiple myeloma and farming. A systematic review of 30 years of research. Where next? J Occup Med Toxicol 2008;3:7.
6. Collotta M, Bertazzi PA, Bollati V. Epigenetics and pesticides. Toxicology 2013;307:35-41.

7. Frost G, Brown T, Harding AH. Mortality and cancer incidence among British agricultural pesticide users. Occup Med 2011;61:303-10.

8. Office for National Statistics. 2011 Census: Key Statistics for Englandand Wales, 2011. 22/02/2016. http://www.ons.gov.uk/ ons/rel/census/2011-census/key-statistics-for-local-authorities-inengland-and-wales/stb-2011-census-key-statistics-for-england-andwales.html.

9. Office for National Statistics. Self-employed workers in the UK, 2014. 22/02/2016. http://www.ons.gov.uk/ons/rel//mac/self-employedworkers-in-the-uk/2014/rep-self-employed-workers-in-the-uk-2014. html.

10. (accessed 11 Jul 2016).

11. Health and Social Care Information Centre. Statistics on Smoking, England, 2013. http://www.hscic.gov.uk/catalogue/PUB11454 (accessed 11/07/2016).

12. Food Standards Agency, Department of Health. National Diet and Nutrition Survey:Headline results from Years 1 and 2 (combined) of the RollingProgramme (2008/09 - 2009/10, 2009. https://www. gov.uk/government/uploads/system/uploads/attachment_data/file/ 216484/dh_128550.pdf (accessed 11 Jul 2016).

13. Holmes EM. Health and Safety Executive. The Pesticide Users' Health Study: Survey of Pesticide Usage, 2013. Research Report RR957. 